\title{
EIGENFUNCTION EXPANSIONS OF ANALYTIC FUNCTIONS
}

\author{
R. T. SEELEY ${ }^{1}$
}

In [5, Theorem 10.2], there was derived a simple result characterizing $C^{\infty}$ sections $f$ of a vector bundle over a compact manifold, in terms of the rate of decay of the coefficients of $f$ in eigenfunctions of a $C^{\infty}$ differential operator. Here we derive a similar result for analytic sections, mentioned in [5]. Following the proof are several applications (the first of which motivates the general proof) and an alternate proof based on a conversation with F. E. Browder.

THEOREM. Let $E$ be a complex vector bundle over the compact realanalytic manifold $X$. Suppose $X$ has an analytic volume element, that $E$ has an analytic Hermitian inner product, and that $A$ is an analytic, elliptic, normal differential operator of order $m$ on the sections of $E$. Let $\left\{\phi_{k}\right\}$ and $\left\{\lambda_{k}\right\}$ be respectively the eigensections and eigenvalues of $A: A \phi_{k}=\lambda_{k} \phi_{k}$, and let $\mu_{k}$ be the positive mth root of $\left|\lambda_{k}\right|$. Then $f=\sum f_{k} \phi_{k}$ is analytic if and only if the sequence $\left\{s^{\mu_{k}}\left|f_{k}\right|\right\}$ is bounded for some $s>1$.

The condition of the theorem is equivalent to: $\sum s^{\mu_{k}}\left|f_{k}\right|^{2}<\infty$ for some $s>1$, as the proof shows.

By normality of $A$ we mean $A^{*} A=A A^{*}$. This guarantees the existence of a basis of orthonormal eigensections, as follows. The null space of $A$ is finite dimensional [5, Theorem 8.3], and if $P$ is orthogonal projection onto this null space, then $P+A$ is normal and has trivial null space and closed range. It follows that $P+A$ is an isomorphism from $H^{m}(E)$ (the space of sections of $E$ all of whose derivatives of order less than $m+1$ are square integrable) onto $H^{0}(E)$, the space of square integrable sections of $E$. Then $P+A$ has an inverse $B$ which is a compact normal operator on $H^{0}(E)$. Since $B$ has orthonormal eigensections $\left\{\phi_{k}\right\}$ with eigenvalues converging to zero, the eigenvalues $\left\{\lambda_{k}\right\}$ of $A$ converge to infinity. More precisely we have

$$
\sum\left|\lambda_{k}\right|^{-2 n}<\infty,
$$

where $n$ is the dimension of $X$. For $\left|\lambda_{k}\right|^{2 n}$ are the eigenvalues of

Received by the editors August 9, 1968 .

1 This paper contains observations made in 1962 while the author was supported by a NATO fellowship at the Mathematisch Centrum, Amsterdam, and written up in 1963 with the support of an NSF research grant, both of which are acknowledged with thanks. 
$\left(A A^{*}\right)^{n}=A^{n}\left(A^{*}\right)^{n}$, while $\left[P+\left(A A^{*}\right)^{n}\right]^{-1}$ is an operator of trace class [5, Lemma 10.1].

Since the $\phi_{k}$ are eigensections of $A^{*} A+I$ with eigenvalues $\left|\lambda_{k}\right|^{2}+1$, we may assume that $A$ is positive, that $\lambda_{k}>0$, and that the order $m$ of $A$ is even.

The proof depends on imbedding $X$ in the open manifold $X^{\prime}$ $=X \times I$, where $I$ is the open interval $(0,2)$, and $X$ is identified with $X \times\{1\}$. We rely on the Cauchy-Kowalewski theorem to derive the rate of decay of the coefficients from the analyticity of $f$, and on the analyticity of solutions of elliptic equations for the converse proof.

Extend the bundle $E$ in the obvious way to $X^{\prime}$, denoting the extension by $E^{\prime}=E \times I$. If $\pi$ is the projection of $E$ onto $X$, then $\pi^{\prime}: E^{\prime} \rightarrow X^{\prime}$ is defined by $\pi^{\prime}(e, t)=(\pi(e), t)$. We consider sections of $E^{\prime}$ as maps $f^{\prime}: X \times I \rightarrow E$ such that $\pi f^{\prime}(x, t)=x$. Define the operator $A^{\prime}$ on sections of $E^{\prime}$ by $A^{\prime} f^{\prime}(x, t)=(A f)(x, t)+i(t \partial / \partial t)^{m} f(x, t)$, where $m$ is the order of $A$. Then $A^{\prime}$ is an analytic differential operator on sections of $E^{\prime}$, and since we have assumed $m$ is even and the characteristic polynomial (symbol) of $A$ is positive definite, it follows that $A^{\prime}$ is elliptic.

Suppose now $f$ is an analytic section of $E$. Then for some $\epsilon>0$, there is an analytic solution $f^{\prime}$ in $X \times[1,1+\epsilon) \subset X^{\prime}$ of the Cauchy problem: $A^{\prime} f^{\prime}=0, f^{\prime}(x, 1)=f(x),(t \partial / \partial t)^{i} f^{\prime}(x, 1)=0$ for $j=1, \cdots, m-1$. Writing $a_{k}(t)=\int_{X}\left\langle f^{\prime}(x, t), \phi_{k}(x)\right\rangle d x$, where $\langle$,$\rangle denotes the Hermitian$ inner product in any fibre of $E$, we have

$$
(t \partial / \partial t)^{m} a_{k}(t)=i \int_{X}\left\langle A f^{\prime}(x, t), \phi_{k}(x)\right\rangle d x=i \lambda_{k} a_{k}(t) .
$$

Thus $a_{k}(t)=\sum_{j=1}^{m} A_{k, j} t^{\theta_{j} \mu_{k}}$, where $\mu_{k}>0,\left(\mu_{k}\right)^{m}=\lambda_{k}$, and $\left\{\theta_{j}\right\}_{1}^{m}$ are the roots of $\theta^{m}=i=\sqrt{ }(-1)$. Applying the data for $t=1$, we find

$$
\sum_{j=1}^{m} A_{k, j}\left(\theta_{j}\right)^{p}=\delta_{\mathrm{op}} f_{k},
$$

where $f_{k}=\int_{X}\left\langle f(x), \phi_{k}(x)\right\rangle d x$. Thus $A_{k, j}=c_{j} f_{k}$, where $\left\{c_{j}\right\}$ is the unique solution of

$$
\sum_{j=1}^{m} c_{j}\left(\theta_{j}\right)^{p}=\delta_{\mathrm{op}}, \quad p=0, \cdots, m-1
$$

Note that the $c_{j}^{\prime}$ 's are quotients of nonvanishing van der Monde determinants and thus no $c_{j}=0$. Now

$$
\int_{X}\left\langle f^{\prime}(x, t), f^{\prime}(x, t)\right\rangle d x=\sum_{k=1}^{\infty}\left|\sum_{j=1}^{m} c_{j} f_{k} t^{\theta_{j} \mu_{k}}\right|^{2}<\infty
$$


for $1 \leqq t<1+\epsilon$, so $\left\{\left|\sum_{1}^{m} c_{j} t^{\theta_{j} \mu_{k}}\right|\left|f_{k}\right|\right\}$ is bounded for some fixed $t>1$. Letting $\theta_{1}=e^{i \pi / 2 m}$, we have $\operatorname{Re}\left(\theta_{1}\right)>\operatorname{Re}\left(\theta_{j}\right)$ for $j=2, \cdots, m$. Since

$$
\sum_{1}^{m} c_{j} t^{\theta_{j} \mu_{k}}=t^{\theta_{1} \mu_{k}}\left(c_{1}+\sum_{2}^{m} c_{j} t^{\left(\theta_{j}-\theta_{1}\right) \mu_{k}}\right)
$$

while $\operatorname{Re}\left(\mu_{k}\left(\theta_{j}-\theta_{1}\right)\right) \rightarrow-\infty$ and $c_{1} \neq 0$, we have that $\left\{\left|t^{\theta_{1} \mu_{k}} f_{k}\right|\right\}$ is bounded. If $\alpha$ is the real part of $\theta_{1}$, and $s=t^{\alpha}$, we then have $s>1$ and $\left\{s^{\mu_{k}}\left|f_{k}\right|\right\}$ is bounded, which proves the first part of the theorem.

For the converse, we construct an $L^{2}$ solution $u$ of $A^{\prime} u=0$ with $u(x, 1)=f(x)$, and then observe that since $A^{\prime}$ is analytic and elliptic, $u$ is analytic $[2, \S 5]$. The construction of $u$ proceeds as follows.

First, from the boundedness of $\left\{s^{\mu_{k}}\left|f_{k}\right|\right\}$ we conclude that $\sum t^{2} \mu_{k}\left|f_{k}\right|^{2}<\infty$ for $0 \leqq t<s$. For if $r=t / s, \quad \sum t^{2 \mu_{k}}\left|f_{k}\right|^{2} \leqq M \sum r^{2 \mu_{k}}$. Since $\sum\left(\mu_{k}\right)^{-p}<\infty$ for an appropriate $p$ (by (1)), $\sum\left|\log r^{2 \mu_{k}}\right|-p<\infty$, and the comparison test shows that $\sum r^{2 \mu_{k}<\infty}$.

Thus writing $u(x, t)=\sum_{1}^{\infty} \sum_{1}^{m} f_{k} c_{j} t^{\theta_{j} \mu_{k}} \phi_{k}(x)$ for $s^{-1}<t<s$ (with $c_{j}$ as in (2) and $\left.\left(\theta_{j}\right)^{m}=i\right)$, we have that $u$ is square integrable on every compact subset of $X \times\left\{s^{-1}<t<s\right\}$. It is also easy to show that for each $C^{\infty}$ section $\psi$ of $E^{\prime}$ with compact support in $X \times\left\{s^{-1}<t<s\right\}$, we have $\left(u,\left(A^{\prime}\right)^{*} \phi\right)=0$, so that $u$ is a "weak" solution of $A^{\prime} u=0$. It follows from standard regularity theorems that $u$ is $C^{\infty}[1$, Theorem 8.1], and then analytic $[2, \S 5]$. Finally, since $f$ is the restriction of $u$ to $X \times\{1\}$, $f$ is analytic.

Applications. If we let $A$ be the Laplace operator on the unit sphere $\{|x|=1\}$ in $R^{n+1}$, then the eigenfunction expansion in question is the spherical harmonic expansion $f(x)=\sum f_{j k} S_{j k}(x)(|x|=1)$ where $S_{j k}$ is a spherical harmonic of degree $j$. The eigenvalues are $\lambda_{j k}$ $-j(j+n-2)$, and $k$ runs from 1 to $(2 j+n-2)(j+n-3) ! / j !(n-2) !$. Thus it follows easily from the general theorem above that $f$ is analytic if and only if $\sum f_{j k} r^{i} S_{j k}$ converges (in $L^{2}$ ) for some $r>1$. Let now Fe be the space of functions harmonic in $\{|x|<1\}$, with the topology of uniform convergence on compact sets; and let $a$ be the set of functions analytic on $\{|x|=1\}$, untopologized. Then we can show immediately that $a$ is the dual of $\mathfrak{F}$. For this, use the base of neighborhoods of zero in $3 C$ given by

$$
U_{r, \delta}=\left\{u \text { in } \mathfrak{H}: \int_{|x|=1}|u(r x)|^{2} d x<\delta\right\} \quad \text { for } 0<r<1, \delta>0 .
$$

Suppose $f^{\wedge}$ is in the dual of $\mathfrak{F}$, let $H_{j k}(x)=|x|{ }^{i} S_{j k}(x /|x|)$, and set 
$f_{j k}=f^{\wedge}\left(H_{j k}\right)$. Suppose $\left|f^{\wedge}(u)\right|<1$ if $u \in U_{r, \delta}$, and let $u=\sum u_{j k} H_{j k}$. Then $\left|\sum f_{j k} u_{j k}\right|<1$ if $\sum\left|u_{j k}\right|^{2} r^{2 j}<\delta$, so $\sum\left|f_{j k} r^{-j}\right|^{2}<\delta^{-1}$, which shows that the $f_{j k}$ are the spherical harmonic coefficients of a function $f$ in a. Conversely, each function in $Q$ leads to a functional on $\mathfrak{H}$, and the isomorphism is established. The same isomorphism can also be realized as follows. Given $f$ analytic on $\{|x|=1\}$, solve the problem (i) $\Delta v(x)=0$ in $|x|>1$, (ii) $v$ bounded in $|x|>1$, (iii) $v(x)=f(x)$ for $|x|=1$. Then $v$ extends analytically to $|x| \geqq r$ for some $r<1$, and for any $u$ in $\mathfrak{H}$ we have $f^{\wedge}(u)=\int_{|x|=1} u(r x) v(r x)$. $a$ can now be given the various topologies of the dual of $\mathfrak{H}$. (For a more general result of this type, see Lions and Magenes [7].)

For a second application, suppose $f$ is analytic in $R^{n+1}-\{0\}$, and for some complex $\lambda, f(t x)=t^{\lambda} f(x)$ for all $t>0$. Then (except for certain integer values of $\lambda$ ), $f$ defines a unique tempered distribution on $\boldsymbol{R}^{n+1}$, which has a fourier transform $f^{\wedge}$. If $f(x)=|x|^{\lambda} \sum f_{j k} S_{j k}(x /|x|)$, then $f^{\wedge}(x)$ comes from the function $|x|^{-\lambda-n-1} \cdot \sum f_{j k} \gamma_{j} S_{j k}(x /|x|)$, with $\gamma_{j}$ $=\pi^{n / 2}(-i)^{i} 2^{\lambda+n} \Gamma((j+n+\lambda) / 2) / \Gamma((j-\lambda) / 2)$ (see [4]). Since $\sum\left|f_{j k}\right|{ }^{2} t^{2 j}$ $<\infty$ for some $t>1$, so is $\sum\left|f_{j k}\right|^{2}\left|\gamma_{j}\right|^{2} s^{2 j}<\infty$ for some $s>1$, and $f$ is analytic. The same result holds, with minor rephrasings, for the exceptional integer values mentioned above.

Another corollary of the expansion theorem is the following: if $B$ is any bounded operator on $H^{0}(E)$ and $A B=B A$, then $B$ maps analytic functions into analytic functions. For if $\left\{\lambda_{j}\right\}$ are the distinct eigenvalues of $A$, and $S_{j}$ is the eigenspace of $\lambda_{j}$, then any $f$ in $H^{0}(E)$ has the expansion $f=\sum a_{j} \phi_{j}$, where $\phi_{j} \in S_{j}$, and $\left\{\phi_{j}\right\}$ extends to an orthonormal basis of eigensections. If $B \phi_{j}=b_{j} \psi_{j}$ with $b_{j}$ complex and $\left\|\psi_{j}\right\|=1$, then $\left|b_{j}\right| \leqq\|B\|, \psi_{j} \in S_{j}$, and $\left\{\psi_{j}\right\}$ extends to an orthonormal basis of eigenfunctions. Since $B f=\sum a_{j} b_{j} \psi_{j}$, we find $B f$ is analytic when $f$ is.

Finally, if $A$ is a positive semidefinite elliptic operator, then for each positive number $L$ and each real number $\alpha$ there is a well defined positive operator $(A+L)^{\alpha}$ on $H^{0}(E)$. It is an easy consequence of the above theorem that, if $A$ is analytic, then $(A+L)^{\alpha}$ maps analytic functions into analytic functions, and in fact the map is continuous and invertible in appropriate topologies. In particular, the operator $\Lambda=(L-\Delta)^{1 / 2}$ constructed in [6] has this property.

Alternate proof. We can also base the proof of our main theorem on a result of Kotake and Narasimhan [3]. This result uses the technique of [2], and applies to an elliptic operator on an arbitrary open set in Euclidean space. We show that our criterion for analyticity is equivalent to the criterion of [3] applied to a compact manifold. Letting 
again $A \phi_{j}=\lambda_{j} \phi_{j}$ and $\mu_{j}^{m}=\left|\lambda_{j}\right|$, the criterion of [3] for analyticity of $f=\sum f_{j} \phi_{j}$ is: there is a constant $C$ such that for all $k \geqq 0$

$$
\sum_{\mu_{j}}^{2 k m}\left|f_{j}\right|^{2} \leqq((k m) !)^{2} C^{2 k+2} \text {. }
$$

The equivalence of (3) with our main theorem reduces easily to the following:

Lemma. Let $0<\mu_{j}<\infty$, and suppose $\sum r^{\mu_{j}}<\infty$ for each $r<1$. Then the condition (3) on sequences $\left\{f_{j}\right\}$ is equivalent to

$$
\left|f_{j}\right| \leqq D t^{\mu_{j}} \text { for some } D<\infty \text { and } t<1 \text {. }
$$

Note that the condition $\sum r^{\mu_{j}}<\infty$ has been derived from (1) in the course of our original proof, when $\left(\mu_{j}\right)^{m}$ is the $j$ th eigenvalue of $A$.

To prove the lemma, assume (3). Then each term of the series in the left of (3) is bounded by the right of (3), so $\left(\mu_{j} / k\right)^{k}\left|f_{j}\right|^{1 / m}$ $\leqq\left(m C^{1 / m}\right)^{k} C^{1 / m}$. Stirling's formula gives, for an appropriate constant $B,\left|f_{j}\right|^{1 / m}\left(\mu_{j}\right)^{k} / k ! \leqq(B / 2)^{k+1}$, so that $\left|f_{j}\right|^{1 / m} \sum\left(\mu_{j} / B\right)^{k} / k ! \leqq B$, i.e. $\left|f_{j}\right| \leqq B^{m}\left(e^{-1 / B}\right)^{\mu_{j}}$, which is (4).

For the converse, we assume (4) and prove $\sum \mu_{j}^{k}\left|f_{j}\right| \leqq k ! C^{k+1}$, which implies (3). Consider $\psi(z)=\sum e^{z \mu_{j}}$. Since $\sum r^{\mu_{j}}<\infty$ for each $r<1, \psi$ is analytic for $\operatorname{Re}(z)<0$, and thus on the compact set $\{z=\log t\}$ satisfies $\left|\psi^{(k)}(z)\right| \leqq k ! C^{k+1}$, where $\psi^{(k)}$ is the $k$ th derivative of $\psi$. Using this, we find $\sum \mu_{j}^{k}\left|f_{j}\right| \leqq D \sum \mu_{j}^{k} t_{j}=D \psi^{(k)}(\log t) \leqq k ! C^{k+1}$.

\section{REFERENCES}

1. L. Hörmander, On interior regularity of the solutions of partial differential equations, Comm. Pure Appl. Math. 11 (1958), 197-218.

2. C. B. Morrey and L. Nirenberg, On the analyticity of the solutions of linear elliptic systems of partial differential equations, Comm. Pure Appl. Math. 10 (1957), 271-290.

3. Takeshi Kotake and M. S. Narasimhan, Regularity theorems for fractional powers of a linear elliptic operator, Bull. Soc. Math. France 90 (1962), 449-471.

4. R. T. Seeley, Homogeneous distributions, Report TW 88, Mathematisch Centrum, Amsterdam.

5. - Integro-differential operators on vector bundles, Trans. Amer. Math. Soc. 117 (1965), 167-204.

6. - Singular integrals on compact manifolds, Amer. J. Math 81 (1959), 658-690.

7. J. L. Lions and E. Magenes, Problemes aux limites non homogenes, Ann. Mat. Pura Appl. 63 (1963), 201-224.

BRANDEIS UNIVERSITY 